\title{
Jet-Ricci Geometry of Time-Dependent Human Biomechanics
}

\author{
Tijana T. Ivancevic \\ Society for Nonlinear Dynamics in Human Factors, Adelaide, Australia \\ and \\ CITECH Research IP Pty Ltd, Adelaide, Australia \\ e-mail: tijana.ivancevic@alumni.adelaide.edu.au
}

\begin{abstract}
We propose the time-dependent generalization of an 'ordinary' autonomous human biomechanics, in which total mechanical + biochemical energy is not conserved. We introduce a general framework for time-dependent biomechanics in terms of jet manifolds derived from the extended musculo-skeletal configuration manifold. The corresponding Riemannian geometrical evolution follows the Ricci flow diffusion. In particular, we show that the exponential-like decay of total biomechanical energy (due to exhaustion of biochemical resources) is closely related to the Ricci flow! on the biomechanical configuration manifold.
\end{abstract}

Keywords: Time-dependent biomechanics, extended configuration manifold, configuration bundle, jet manifolds, Ricci flow diffusion

\section{Introduction}

It is a well-known fact that most of Hamiltonian and Lagrangian dynamics in physics are based on assumption of a total energy conservation. Even more, the word "Hamiltonian" usually means "conservative", while Lagrangian formalism is usually proved to be equivalent to Hamiltonian (therefore also conservative), as derived from a conservative Lagrangian energy function (for a comprehensive review see, e.g. 1]). The straightforward application of Hamiltonian/Lagrangian formalisms to human biomechanics would naturally inherit this conservative assumption 2

And this works fine for most individual movement simulations and predictions, in which the total human energy dissipations are insignificant. However, if we analyze a $100 \mathrm{~m}$-dash sprinting motion, which is in case of top athletes finished under $10 \mathrm{~s}$, we can recognize a significant slow-down after about $70 \mathrm{~m}$ in all athletes - despite of their strong intention to

\footnotetext{
${ }^{1}$ Ricci flow is a current hot topic in pure mathematics for which the latest Fields Medal was awarded to G. Perelman for the proof of 100 year-old Poincaré Conjecture.

${ }^{2}$ An engineering-type approach to this problem would be simply adding dissipation and forcing into equations of motion, without deriving them form some "dissipative Lagrangian" and/or "dissipative Hamiltonian"; this yields an extended Hamiltonian and/or Lagrangian formalism (see [2, 3, 4, 5, 6, 7, 8, 9]).
} 
finish and win the race, which is an obvious sign of the total energy dissipation. This can be seen, for example, in a current record-braking speeddistance curve of Usain Bolt, the world-record holder with $9.69 \mathrm{~s}$ [10], or in a former record-braking speeddistance curve of Carl Lewis, the former world-record holder (and 9 time Olympic gold medalist) with 9.86 s (see Figure 3.7 in [9]). In other words, the total mechanical + biochemical energy of a sprinter cannot be conserved even for $10 \mathrm{~s}$. So, if we want to develop a realistic model of intensive human motion that is longer than $7-8 \mathrm{~s}$ (not to speak for instance of a 4 hour tennis match), we necessarily need to use the more advanced formalism of time-dependent mechanics.

Similarly, if we analyze individual movements of gymnasts or pirouettes in ice skating, we can clearly see that the high speed of these movements is based on quickly-varying mass-inertia distribution of various body segments (mostly arms and legs). As the total mass-inertia matrix of a biomechanical system corresponds to the Riemannian metric tensor of its configuration manifold, we can formulate this problem in terms of time-dependent Riemannian geometry [4, 1].

The purpose of this paper is to introduce a general framework for time-dependent biomechanics, consisting of:

1. human biomechanical configuration manifold and its (co)tangent bundles;

2. biomechanical jet spaces and prolongation of locomotion vector-fields developed on the biomechanical configuration manifold; and

3. time-dependent Lagrangian dynamics using biomechanical jet spaces.

In addition, we will show that Riemannian geometrical basis of this framework is defined by the Ricci flow. In particular, we will show that the exponential-like decay of total biomechanical energy (due to exhaustion of biochemical resources [9]) is closely related to the Ricci flow on the configuration manifold of human motion.

\section{Human Biomechanical Manifold and its (Co)Tangent Bundles}

\subsection{Humanoid Robot Dynamics}

Recall from [6] that representation of an ideal humanoid-robot motion is rigorously defined in terms of rotational constrained $S O(3)$-groups in all main robot joints. Therefore, the configuration manifold $Q_{r o b}$ for humanoid dynamics is defined as a topological product of all included $S O(3)$ groups, $Q_{r o b}=\prod_{i} S O(3)^{i}$. Consequently, the natural stage for autonomous Lagrangian dynamics of robot motion is the tangent bundle $T Q_{r o b}$, defined as follows. To each $n$-dimensional $(n \mathrm{D})$ configuration manifold $Q$ there is associated its $2 n \mathrm{D}$ velocity phase-space manifold, denoted by $T Q$ and called the tangent bundle of $Q$. The original smooth manifold $Q$ is called the base of $T Q$. There is an onto map $\pi: T Q \rightarrow Q$, called the projection. Above each point $x \in Q$ there is a tangent space $T_{x} Q=\pi^{-1}(x)$ to $Q$ at $x$, which is called a fibre. The fibre $T_{x} Q \subset T Q$ is the subset of $T Q$, such that the total tangent bundle, $T Q=\bigsqcup_{m \in Q} T_{x} Q$, is a disjoint union of tangent spaces $T_{x} Q$ to $Q$ for all 
points $x \in Q$. From dynamical perspective, the most important quantity in the tangent bundle concept is the smooth map $v: Q \rightarrow T Q$, which is an inverse to the projection $\pi$, i.e, $\pi \circ v=\operatorname{Id}_{Q}, \pi(v(x))=x$. It is called the velocity vector-field. Its graph $(x, v(x))$ represents the cross-section of the tangent bundle $T Q$. This explains the dynamical term velocity phase-space, given to the tangent bundle $T Q$ of the manifold $Q$. The tangent bundle is where tangent vectors live, and is itself a smooth manifold. Vector-fields are cross-sections of the tangent bundle. Robot's Lagrangian (energy function) is a natural energy function on the tangent bundle $T Q 3$

\subsection{Realistic Human Configuration Manifold}

On the other hand, human joints are more flexible than robot joints. Namely, every rotation in all synovial human joints is followed by the corresponding micro-translation, which occurs after the rotational amplitude is reached [6. So, representation of human motion is rigorously defined in terms of Euclidean $S E(3)$-groups of full rigid-body motion [11, 2, 4, 1] in all main human joints (see Figure 1). Therefore, the configuration manifold $Q$ for human dynamics is defined as a topological product of all included constrained $S E(3)$ groups, $Q=\prod_{i} S E(3)^{i}$. Consequently, the natural stage for autonomous Lagrangian dynamics of human motion is the tangent bundle $T Q$ (and for the corresponding autonomous Hamiltonian dynamics is the cotangent bundle $T^{*} Q$ ).

Briefly, the Euclidean SE(3)-group is defined as a semidirect (noncommutative) product (denoted by $\triangleright$ ) of $3 \mathrm{D}$ rotations and $3 \mathrm{D}$ translations: $S E(3):=S O(3) \triangleright \mathbb{R}^{3}$. Its most important subgroups are the following (for technical details see [4, 12, 1]):

\begin{tabular}{|cc|}
\hline Subgroup & Definition \\
\hline$S O(3)$, group of rotations & Set of all proper orthogonal \\
in 3D (a spherical joint) & $3 \times 3-$ rotational matrices \\
\hline $\begin{array}{c}S E(2), \text { special Euclidean group } \\
\text { in 2D (all planar motions) }\end{array}$ & Set of all $3 \times 3-$ matrices: \\
& {$\left[\begin{array}{ccc}\cos \theta & \sin \theta & r_{x} \\
-\sin \theta & \cos \theta & r_{y} \\
0 & 0 & 1\end{array}\right]$} \\
\hline $\begin{array}{c}S O(2), \text { group of rotations in 2D } \\
\text { subgroup of } S E(2)-\text { group } \\
\text { (a revolute joint) }\end{array}$ & $\begin{array}{c}\text { Set of all proper orthogonal } \\
\text { included in } S E(2)-\text { group }\end{array}$ \\
\hline $\mathbb{R}^{3}, \begin{array}{l}\text { group of translations in 3D } \\
\text { (all spatial displacements) }\end{array}$ & Euclidean 3D vector space \\
\hline
\end{tabular}

The configuration manifold $Q=\prod_{i} S E(3)^{i}$ has the Riemannian geometry with the local

${ }^{3}$ The corresponding autonomous Hamiltonian robot dynamics takes place in the cotangent bundle $T^{*} Q_{r o b}$, defined as follows. A dual notion to the tangent space $T_{m} Q$ to a smooth manifold $Q$ at a point $m$ is its cotangent space $T_{m}^{*} Q$ at the same point $m$. Similarly to the tangent bundle, for a smooth manifold $Q$ of dimension $n$, its cotangent bundle $T^{*} Q$ is the disjoint union of all its cotangent spaces $T_{m}^{*} Q$ at all points $m \in Q$, i.e., $T^{*} Q=\bigsqcup_{m \in Q} T_{m}^{*} Q$. Therefore, the cotangent bundle of an $n$-manifold $Q$ is the vector bundle $T^{*} Q=(T Q)^{*}$, the (real) dual of the tangent bundle $T Q$. The cotangent bundle is where 1 -forms live, and is itself a smooth manifold. Covector-fields (1-forms) are cross-sections of the cotangent bundle. Robot's Hamiltonian is a natural energy function on the cotangent bundle. 


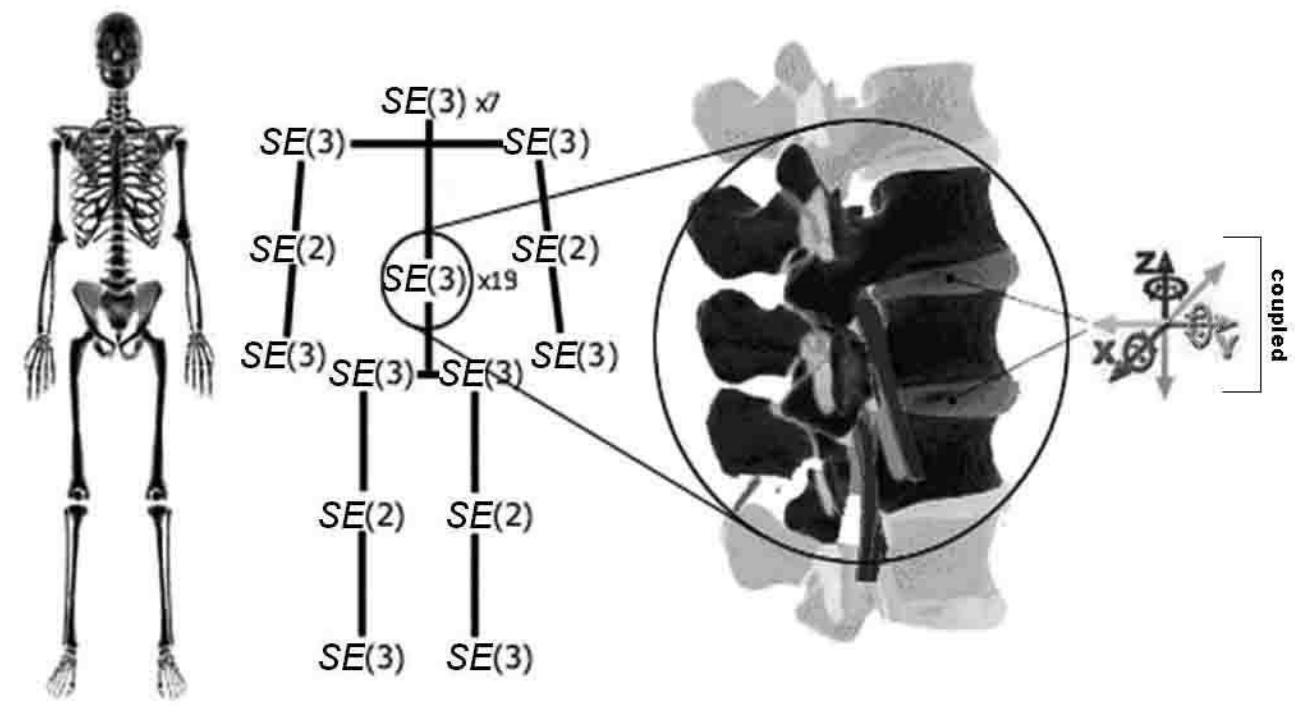

Figure 1: The configuration manifold $Q$ of the human musculo-skeletal dynamics is defined as a topological product of constrained $S E(3)$ groups acting in all major (synovial) human joints, $Q=\prod_{i} S E(3)^{i}$. The manifold

metric form:

$$
\langle g\rangle \equiv d s^{2}=g_{i j} d x^{i} d x^{j}, \quad \text { (Einstein's summation convention is in use) }
$$

where $g_{i j}(x)$ is the material metric tensor defined by the biomechanical system's massinertia matrix and $d x^{i}$ are differentials of the local joint coordinates $x^{i}$ on $Q$. Besides giving the local distances between the points on the manifold $Q$, the Riemannian metric form $\langle g\rangle$ defines the system's kinetic energy:

$$
T=\frac{1}{2} g_{i j} \dot{x}^{i} \dot{x}^{j},
$$

giving the Lagrangian equations of the conservative skeleton motion with kinetic-minuspotential energy Lagrangian $L=T-V$, with the corresponding geodesic form [8]

$$
\frac{d}{d t} L_{\dot{x}^{i}}-L_{x^{i}}=0 \quad \text { or } \quad \ddot{x}^{i}+\Gamma_{j k}^{i} \dot{x}^{j} \dot{x}^{k}=0,
$$

where subscripts denote partial derivatives, while $\Gamma_{j k}^{i}$ are the Christoffel symbols of the affine Levi-Civita connection of the biomechanical manifold $Q$.

This is the basic geometrical structure for autonomous Lagrangian biomechanics. In the next section will extend this basic structure to embrace the time-dependent biomechanics.

\section{Biomechanical Jet Spaces}

In general, tangent and cotangent bundles, $T M$ and $T^{*} M$, of a smooth manifold $M$, are special cases of a more general geometrical object called fibre bundle, where the word fiber $V$ 

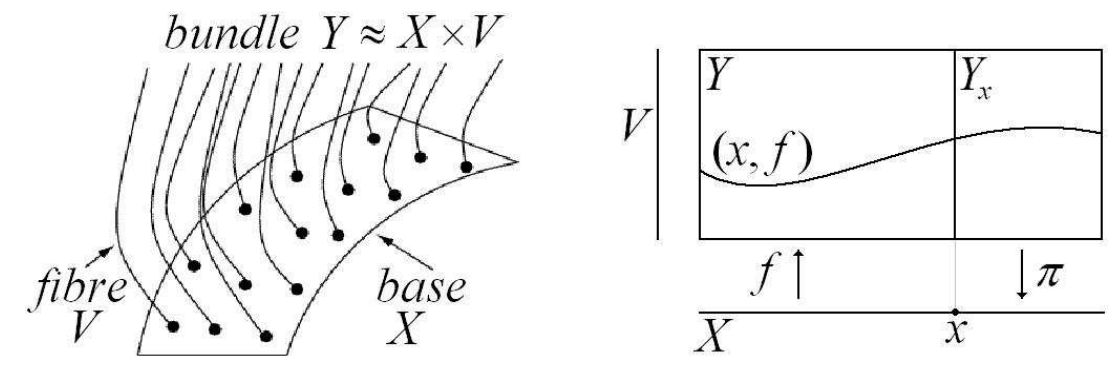

Figure 2: A sketch of a fibre bundle $Y \approx X \times V$ as a generalization of a product space $X \times V$; left - main components; right - a few details (see text for explanation).

of a map $\pi: Y \rightarrow X$ denotes the preimage $\pi^{-1}(x)$ of an element $x \in X$. It is a space which locally looks like a product of two spaces (similarly as a manifold locally looks like Euclidean space), but may possess a different global structure. To get a visual intuition behind this fundamental geometrical concept, we can say that a fibre bundle $Y$ is a homeomorphic generalization of a product space $X \times V$ (see Figure 2), where $X$ and $V$ are called the base and the fibre, respectively. $\pi: Y \rightarrow X$ is called the projection, $Y_{x}=\pi^{-1}(x)$ denotes a fibre over a point $x$ of the base $X$, while the map $f=\pi^{-1}: X \rightarrow Y$ defines the cross-section, producing the graph $(x, f(x))$ in the bundle $Y$ (e.g., in case of a tangent bundle, $f=\dot{x}$ represents a velocity vector-field).

The main reason why we need to study fibre bundles is that all dynamical objects (including vectors, tensors, differential forms and gauge potentials) are their cross-sections, representing generalizations of graphs of continuous functions.

By extending this line of formal bundle thinking, we come to the concept of a jet manifold, which is based on the idea of higher-order tangency, or higher-order contact, at some designated point on a smooth manifold. Namely, a pair of smooth manifold maps, $f_{1}, f_{2}: M \rightarrow N$ (see Figure 3), are said to be $k$-tangent (or tangent of order $k$, or have a $k$ th order contact) at a point $x$ on a domain manifold $M$, denoted by $f_{1} \sim f_{2}$, iff

$$
\begin{aligned}
f_{1}(x)= & f_{2}(x) \quad \text { called } \quad 0-\text { tangent, } \\
\partial_{x} f_{1}(x)= & \partial_{x} f_{2}(x), \quad \text { called } 1-\text { tangent, } \\
\partial_{x x} f_{1}(x)= & \partial_{x x} f_{2}(x), \quad \text { called } \quad 2-\text { tangent, } \\
& \ldots \quad \text { etc. to the order } k
\end{aligned}
$$

In this way defined $k$-tangency is an equivalence relation, i.e.,

$$
f_{1} \sim f_{2} \Rightarrow f_{2} \sim f_{1}, \quad f_{1} \sim f_{2} \sim f_{3} \Rightarrow f_{1} \sim f_{3}, \quad f_{1} \sim f_{1} .
$$

Now, a $k$-jet (or, a jet of order $k$ ), denoted by $j_{x}^{k} f$, of a smooth map $f: M \rightarrow N$ at a point $x \in M$ (see Figure 3), is defined as an equivalence class of $k$-tangent maps at $x$,

$$
j_{x}^{k} f: M \rightarrow N=\left\{f^{\prime}: f^{\prime} \text { is } k \text { - tangent to } f \text { at } x\right\}
$$

In the same way as in the case of a map $f: M \rightarrow N, x \mapsto f(x)$, also in the case of the $k$-jet $j_{x}^{k} f: M \rightarrow N, x \mapsto f(x)$, the point $x$ in the domain $M$ is called the source of $j_{x}^{k} f$ and the point $f(x)$ in the codomain $N$ is the target of $j_{x}^{k} f$. 


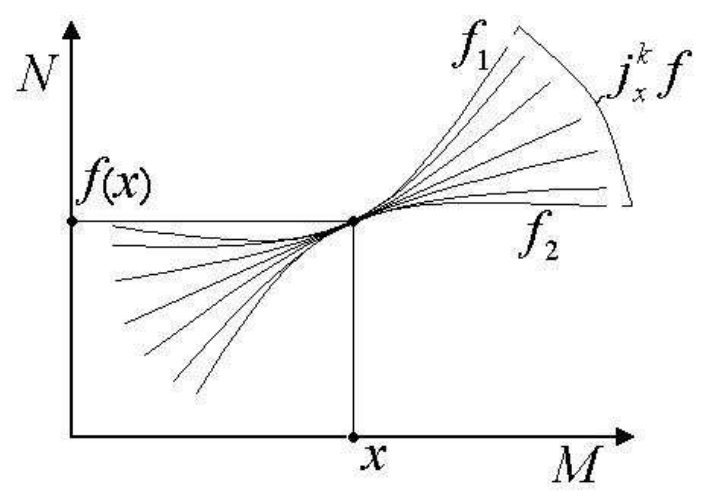

Figure 3: An intuitive geometrical picture behind the $k$-jet concept, based on the idea of a higher-order tangency (or, higher-order contact).

We choose local coordinates on $M$ and $N$ in the neighborhood of the points $x$ and $f(x)$, respectively. Then the $k$-jet $j_{x}^{k} f$ of any map close to $f$, at any point close to $x$, can be given by its Taylor-series expansion at $x$, with coefficients up to degree $k$. Therefore, in a fixed coordinate chart, the $k$-jet is given by:

$$
j_{x}^{k} f: M \rightarrow N \equiv\{\text { collection of Taylor coefficients up to degree } k\} .
$$

The set of all $k$-jets from $M$ to $N$ is called the $k$-jet space $J^{k}(M, N)$. It has a natural smooth-manifold structure. Also, a map from a $k$-jet manifold $J^{k}(M, N)$ to a smooth manifold $M$ or $N$ is called a $k$-jet bundle.

For example, consider a simple function $f: X \rightarrow Y, x \mapsto y=f(x)$, mapping the $X$-axis into the $Y$-axis in $\mathbb{R}^{2}$. In this case, $X$ is a domain and $Y$ is a codomain. At a chosen point $x \in X$ we have:

a 0 -jet is a graph: $(x, f(x))$;

a $1-$ jet is a triple: $\left(x, f(x), f^{\prime}(x)\right)$;

a 2 -jet is a quadruple: $\left(x, f(x), f^{\prime}(x), f^{\prime \prime}(x)\right)$,

and so on, up to the order $k$ (where $f^{\prime}(x)=\frac{d f(x)}{d x}$, etc).

The set of all $k$-jets from $j_{x}^{k} f: X \rightarrow Y$ is called the $k$-jet manifold $J^{k}(X, Y)$.

We now turn back into the field of time-dependent human biomechanics, where the fundamental geometrical construct is the configuration fibre bundle $\pi: Q \rightarrow \mathbb{R}$. Given a configuration fibre bundle $Q \rightarrow \mathbb{R}$ over the time axis $\mathbb{R}$, we say that the 1 -jet manifold $J^{1}(\mathbb{R}, Q)$ is the set of equivalence classes $j_{t}^{1} s$ of sections $s^{i}: \mathbb{R} \rightarrow Q$ of the bundle $Q \rightarrow \mathbb{R}$, which are identified by their values $s^{i}(t)$, as well as by the values of their partial derivatives $\partial_{t} s^{i}=\partial_{t} s^{i}(t)$ at time points $t \in \mathbb{R}$. The 1 -jet manifold $J^{1}(\mathbb{R}, Q)$ is coordinated by $\left(t, x^{i}, \dot{x}^{i}\right)$, so the 1 -jets are local coordinate maps

$$
j_{t}^{1} s: \mathbb{R} \rightarrow Q, \quad t \mapsto\left(t, x^{i}, \dot{x}^{i}\right)
$$

Similarly, the 2-jet manifold $J^{2}(\mathbb{R}, Q)$ is the set of equivalence classes $j_{t}^{2} s$ of sections $s^{i}$ : $\mathbb{R} \rightarrow Q$ of the configuration bundle $\pi: Q \rightarrow \mathbb{R}$, which are identified by their values $s^{i}(t)$, 
as well as the values of their first and second partial derivatives, $\partial_{t} s^{i}=\partial_{t} s^{i}(t)$ and $\partial_{t t} s^{i}=$ $\partial_{t t} s^{i}(t)$, respectively, at time points $t \in \mathbb{R}$. The 2 -jet manifold $J^{2}(\mathbb{R}, Q)$ is coordinated by $\left(t, x^{i}, \dot{x}^{i}, \ddot{x}^{i}\right)$, so the 2 -jets are local coordinate maps

$$
j_{t}^{2} s: \mathbb{R} \rightarrow Q, \quad t \mapsto\left(t, x^{i}, \dot{x}^{i}, \ddot{x}^{i}\right) .
$$
4.

This global geometrical structure of time-dependent biomechanics is depicted in Figure

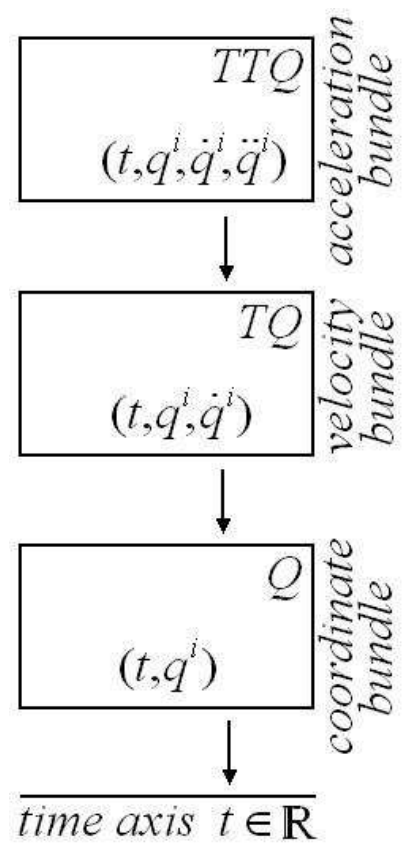

Figure 4: Hierarchical geometrical structure of time-dependent biomechanics. Here, generalized coordinates $q^{i}$, velocities $\dot{q}^{i}$ and accelerations $\ddot{q}^{i}$ replace the corresponding rotational and translational joint coordinates $x^{i}$, velocities $\dot{x}^{i}$ and accelerations $\ddot{x}^{i}$.

In the framework of biomechanics, we consider a pair of maps $f_{1}, f_{2}: \mathbb{R} \rightarrow Q$ from the real line $\mathbb{R}$, representing the time $t$-axis, into a smooth $n$-dimensional Riemannian configuration manifold $Q$ from Figure 1. We say that the two maps $f_{1}=f_{1}(t)$ and $f_{2}=f_{2}(t)$ have the same $k$-jet $j_{t}^{k} f$ at a specified time instant $t_{0} \in \mathbb{R}$, iff:

1. $f_{1}(t)=f_{2}(t)$ at $t_{0} \in \mathbb{R} ;$ and also

2. the first $k$ terms of their Taylor-series expansions around $t_{0} \in \mathbb{R}$ are equal.

The set of all $k$-jets $j_{t}^{k} f: \mathbb{R} \rightarrow Q$ is the $k$-jet manifold $J^{k}(\mathbb{R}, Q)$. In particular, $J^{1}(\mathbb{R}, Q) \cong$ $\mathbb{R} \times T Q$. 


\subsection{Jet Prolongation of Locomotion Vector-fields}

Consider an arbitrary locomotion vector-field $u$ that is on the biomechanical configuration bundle $\pi: Q \rightarrow \mathbb{R}$ defined by

$$
u=u^{t} \partial_{t}+u^{i}\left(t, q^{j}\right) \partial_{i} .
$$

The so-called jet prolongation of the locomotion vector-field $u$ onto the extended velocity phase-space, tht is the 1 -jet manifold $J^{1}(\mathbb{R}, Q)$, reads

$$
J^{1} u=u^{t} \partial_{t}+u^{i} \partial_{i}+d_{t} u^{i} \partial_{i}^{t}
$$

For example, consider a one-dimensional motion of a point particle subject to friction. It is described by the dynamic equation

$$
\ddot{q}=-k \dot{q}, \quad(\text { with } k>0) .
$$

This is the Lagrangian equation for the Lagrangian function

$$
L=\frac{1}{2} \exp [k t] \dot{q}^{2} d t
$$

Let us consider the vector field

$$
v=\partial_{t}-\frac{k}{2} q \partial_{q}
$$

Its jet prolongation $J^{1} v$ reads

$$
J^{1} v=\partial_{t}-\frac{k}{2} q \partial_{q}-\frac{k}{2} q_{t} \partial_{q}^{t}
$$

Generalization of all above jet structures to the $k$-jet manifold $J^{k}(\mathbb{R}, Q)$ is obvious,

$$
j_{t}^{k} s: \mathbb{R} \rightarrow Q, \quad t \mapsto\left(t, x^{i}, \dot{x}^{i}, \ddot{x}^{i}, \dddot{x}^{i}, \ldots, x^{(k) i}\right) .
$$

For more technical details on jet manifolds and bundles with their applications to mechanics and physics, see [18, 19, 20, 21, 22]).

\section{Lagrangian Form of Time-Dependent Biomechanics}

The general form of time-dependent Lagrangian biomechanics with time-dependent Lagrangian function $L\left(t ; x^{i} ; \dot{x}^{i}\right)$ defined on the 1 -jet manifold $X=J^{1}(\mathbb{R}, Q) \cong \mathbb{R} \times T Q$, with local canonical coordinates: $\left(t ; x^{i} ; \dot{x}^{i}\right)=$ (time, coordinates and velocities) in active human joints, can be formulated as [4, 1]

$$
\frac{d}{d t} L_{\dot{x}^{i}}-L_{x^{i}}=\mathcal{F}_{i}(t, x, \dot{x}), \quad(i=1, \ldots, n),
$$

where the coordinate and velocity partial derivatives of the Lagrangian are respectively denoted by $L_{x^{i}}$ and $L_{\dot{x}^{i}}$. The right-hand side terms $\mathcal{F}_{i}(t, x, \dot{x})$ of (2) denote any type of external torques and forces, including [2, 3]: (i) excitation and contraction dynamics of muscular-actuators; (ii) autogenetic (spinal) reflex force controllers; (iii) rotational dynamics of hybrid robot actuators; (iv) (nonlinear) dissipative joint torques and forces; and (v) external stochastic perturbation torques and forces. 


\subsection{Time-Dependent Riemannian Geometry and Ricci Flow}

In the geodesic framework (1), the (in)stability of the biomechanical joint and center-ofmass trajectories is the (in)stability of the geodesics, and it is completely determined by the curvature properties of the underlying manifold according to the Jacobi equation of geodesic deviation [4, 1 ]

$$
\frac{D^{2} J^{i}}{d s^{2}}+R_{j k m}^{i} \frac{d x^{j}}{d s} J^{k} \frac{d x^{m}}{d s}=0,
$$

whose solution $J$, usually called Jacobi variation field, locally measures the distance between nearby geodesics; $D / d s$ stands for the covariant derivative along a geodesic and $R^{i}{ }_{j k m}$ are the components of the Riemann curvature tensor.

On the other hand, the mass-inertia matrix of human body segments, defining the Riemannian metric tensor $g_{i j}=g_{i j}(x)$, need not be time-constant, that is, in general we have $g_{i j}=g_{i j}(x)=g_{i j}(t, x)$. Majority of fast movements in gymnastics are based on fast-changing mass distribution. This time-dependent Riemannian geometry can be formalized in terms of the Ricci flow equation (or, the parabolic Einstein equation), introduced by R. Hamilton in 1982 [24, that is the nonlinear heat-like evolution equation 4

$$
\partial_{t} g_{i j}=-2 R_{i j},
$$

for a time-dependent Riemannian metric $g=g_{i j}(t)$ on a smooth $n$-manifold $Q$ with the Ricci curvature tensor $R_{i j}$. This equation roughly says that we can deform any metric on a 2-surface or $n$-manifold by the negative of its curvature; after normalization, the final state of such deformation will be a metric with constant curvature. The factor of 2 in (3) is more-or-less arbitrary, but the negative sign is essential to insure a kind of global volume exponential decay 5 since the Ricci flow equation (3) is a kind of nonlinear geometric generalization of the standard linear heat equation

$$
\partial_{t} u=\Delta u
$$

Like the heat equation (4), the Ricci flow equation (3) is well behaved in forward time and acts as a kind of smoothing operator (but is usually impossible to solve in backward time).

\footnotetext{
${ }^{4}$ The current hot topic in geometric topology is the Ricci flow, a Riemannian evolution machinery that recently allowed G. Perelman to prove the celebrated Poincaré Conjecture, a century-old mathematics problem and win him the 2006 Fields Medal (which he declined in a public controversy). The Poincaré Conjecture can roughly be put as a question: Is a closed 3-manifold $Q$ topologically a sphere if every closed curve in $Q$ can be shrunk continuously to a point? In other words, Poincaré conjectured: A simply-connected compact 3-manifold is diffeomorphic to the 3-sphere $S^{3}$.

${ }^{5}$ This complex geometric process is globally similar to a generic exponential decay ODE:$$
\dot{x}=-\lambda f(x) \text {, }
$$

for a positive function $f(x)$. We can get some insight into its solution from the simple exponential decay ODE,

$$
\dot{x}=-\lambda x \quad \text { with the solution } \quad x(t)=x_{0} \mathrm{e}^{-\lambda t},
$$

(where $x=x(t)$ is the observed quantity with its initial value $x_{0}$ and $\lambda$ is a positive decay constant), as well as the corresponding $n$th order rate equation (where $n>1$ is an integer),

$$
\dot{x}=-\lambda x^{n} \quad \text { with the solution } \quad \frac{1}{x^{n-1}}=\frac{1}{x_{0}{ }^{n-1}}+(n-1) \lambda t .
$$
}


If some parts of a solid object are hot and others are cold, then, under the heat equation, heat will flow from hot to cold, so that the object gradually attains a uniform temperature. To some extent the Ricci flow behaves similarly, so that the Ricci curvature 'tries' to become more uniform [29, thus resembling a monotonic entropy growth $6 \partial_{t} S \geq 0$, which is due to the positive definiteness of the metric $g_{i j} \geq 0$, and naturally implying the arrow of time [1].

In a suitable local coordinate system, the Ricci flow equation (3) on a biomechanical configuration manifold $Q$ has a nonlinear heat-type form, as follows. At any time $t$, we can choose local harmonic coordinates so that the coordinate functions are locally defined harmonic functions in the metric $g(t)$. Then the Ricci flow takes the general form [31]

$$
\begin{aligned}
\partial_{t} g_{i j} & =\Delta_{Q} g_{i j}+G_{i j}(g, \partial g), \quad \text { where } \\
\Delta_{Q} & \equiv \frac{1}{\sqrt{\operatorname{det}(g)}} \frac{\partial}{\partial x^{i}}\left(\sqrt{\operatorname{det}(g)} g^{i j} \frac{\partial}{\partial x^{j}}\right)
\end{aligned}
$$

is the Laplace-Beltrami operator of the configuration manifold $Q$ and $G_{i j}(g, \partial g)$ is a lowerorder term quadratic in $g$ and its first order partial derivatives $\partial g$. From the analysis of nonlinear heat PDEs, one obtains existence and uniqueness of forward-time solutions to the Ricci flow on some time interval, starting at any smooth initial metric $g_{0}$ on $Q$.

The exponentially-decaying geometrical diffusion (5) is a formal description for pirouettes in ice skating and fast rotational movements in gymnastics.

\subsection{Topological Lagrangian Dynamics}

The biomechanical jet space $X=J^{1}(\mathbb{R}, X) \cong \mathbb{R} \times T Q$ gives rise to the fundamental $n$-groupoid, or $n$-category $\Pi_{n}(X)$ (see [4, 1]). In $\Pi_{n}(X), 0$-cells are points in $X ; 1-$ cells are paths in $X$ (i.e., parameterized smooth maps $f:[0,1] \rightarrow X) ; 2$-cells are smooth homotopies (denoted by $\simeq$ ) of paths relative to endpoints (i.e., parameterized smooth maps $h:[0,1] \times[0,1] \rightarrow X) ; 3$-cells are smooth homotopies of homotopies of paths in $X$ (i.e., parameterized smooth maps $j:[0,1] \times[0,1] \times[0,1] \rightarrow X)$. Categorical composition is defined by pasting paths and homotopies. In this way, the following recursive Lagrangian homotopy

\footnotetext{
${ }^{6}$ Note that two different kinds of entropy functional have been introduced into the theory of the Ricci flow, both motivated by concepts of entropy in thermodynamics, statistical mechanics and information theory. One is Hamilton's entropy, the other is Perelman's entropy. While in Hamilton's entropy, the scalar curvature $R$ of the metric $g_{i j}$ is viewed as the leading quantity of the system and plays the role of a probability density, in Perelman's entropy the leading quantity describing the system is the metric $g_{i j}$ itself. Hamilton established the monotonicity of his entropy along the volume-normalized Ricci flow on the 2-sphere $S^{2}[26$. Perelman established the monotonicity of his entropy along the Ricci flow in all dimensions [30].
} 
dynamics emerges on the configuration manifold $X$ :

$$
\begin{aligned}
& 0-\operatorname{cell}: x_{0} \bullet \quad x_{0} \in X ; \quad \text { in the higher cells below: } t, s \in[0,1] ; \\
& 1-\operatorname{cell}: x_{0} \bullet \stackrel{L}{\longrightarrow} \bullet x_{1} \quad L: x_{0} \simeq x_{1} \in X, \\
& L:[0,1] \rightarrow X, L: x_{0} \mapsto x_{1}, x_{1}=L\left(x_{0}\right), L(0)=x_{0}, L(1)=x_{1} ; \\
& \text { e.g., linear path: } \quad L(t)=(1-t) x_{0}+t x_{1} ; \quad \text { or } \\
& \text { Lagrangian } L-\text { dynamics with endpoint conditions }\left(x_{0}, x_{1}\right):
\end{aligned}
$$$$
\frac{d}{d t} L_{\dot{x}^{i}}=L_{x^{i}}, \quad \text { with } \quad x(0)=x_{0}, \quad x(1)=x_{1}, \quad(i=1, \ldots, n) ;
$$$$
2-\operatorname{cell}: x_{0} \bullet \overbrace{L^{2}}^{L^{1} h} \cdot x_{1} \quad h: L^{1} \simeq L^{2} \in X \text {, }
$$

$h:[0,1] \times[0,1] \rightarrow X, h: L^{1} \mapsto L^{2}, L^{2}=h\left(L^{1}\left(x_{0}\right)\right)$,

$h\left(x_{0}, 0\right)=L^{1}\left(x_{0}\right), h\left(x_{0}, 1\right)=L^{2}\left(x_{0}\right), h(0, t)=x_{0}, h(1, t)=x_{1}$

e.g., linear homotopy: $h\left(x_{0}, t\right)=(1-t) L\left(x_{0}\right)+t L^{2}\left(x_{0}\right)$; $\quad$ or

homotopy between two Lagrangian $\left(L^{1}, L^{2}\right)$ - dynamics

with the same endpoint conditions $\left(x_{0}, x_{1}\right)$ :

$\frac{d}{d t} L_{\dot{x}^{i}}^{1}=L_{x^{i}}^{1}, \quad$ and $\quad \frac{d}{d t} L_{\dot{x}^{i}}^{2}=L_{x^{i}}^{2} \quad$ with $\quad x(0)=x_{0}, \quad x(1)=x_{1} ;$

- etc.

\section{Conclusion}

In this paper we have presented time-dependent generalization of an 'ordinary' autonomous human musculo-skeletal biomechanics. Firstly, we have defined the basic configuration manifold $Q$ of human musculo-skeletal biomechanics as an anthropomorphic chain of constrained Euclidean motion groups $S E(3)$. Secondly, we have extended this base manifold by the real time axis $\mathbb{R}$ into the biomechanical configuration bundle $Q \rightarrow \mathbb{R}$. The time-dependent Lagrangian biomechanics is defined on $Q \rightarrow \mathbb{R}$ using the formalism of first and second order jet manifolds as well as jet prolongations. Then we moved to time-dependent Riemannian geometry, governed by the Ricci-flow diffusion and showed that the exponential-like decay of total biomechanical energy (due to exhaustion of biochemical resources) is closely related to the Ricci-flow based geometrical diffusion.

\section{References}

[1] Ivancevic, V., Ivancevic, T., Applied Differential Geometry: A Modern Introduction. World Scientific, Singapore, (2007)

[2] Ivancevic, V., Ivancevic, T., Human-Like Biomechanics: A Unified Mathematical Approach to Human Biomechanics and Humanoid Robotics. Springer, Dordrecht, (2006) 
[3] Ivancevic, V., Ivancevic, T., Natural Biodynamics. World Scientific, Singapore (2006)

[4] Ivancevic, V., Ivancevic, T., Geometrical Dynamics of Complex Systems: A Unified Modelling Approach to Physics, Control, Biomechanics, Neurodynamics and PsychoSocio-Economical Dynamics. Springer, Dordrecht, (2006)

[5] Ivancevic, V., Ivancevic, T., High-Dimensional Chaotic and Attractor Systems. Springer, Berlin, (2006)

[6] Ivancevic, V., Ivancevic, T., Human versus humanoid robot biodynamcis. Int. J. Hum. Rob. 5(4), 699-713, (2008)

[7] Ivancevic, T., Jovanovic, B., Djukic, M., Markovic, S., Djukic, N., Biomechanical Analysis of Shots and Ball Motion in Tennis and the Analogy with Handball Throws, J. Facta Universitatis, Series: Sport, 6(1), 51-66, (2008)

[8] Ivancevic, T., Jain, L., Pattison, J., Hariz, A., Nonlinear Dynamics and Chaos Methods in Neurodynamics and Complex Data Analysis, Nonl. Dyn. (Springer), 56(1-2), 23-44, (2009)

[9] Ivancevic, T., Jovanovic, B., Djukic, S., Djukic, M., Markovic, S., Complex Sports Biodynamics: With Practical Applications in Tennis, Springer, Berlin, (2009)

[10] Tucker, R., Dugas, J.: Beijing 2008: Men $100 \mathrm{~m}$ race analysis. Bolt's 9.69s. Analysis of speed during the world record. The Science of Sport, http://www.sportsscientists.com/2008/08/beijing-2008-men-100m-race-analysis.html, (2008)

[11] Marsden, J.E., Ratiu, T.S., Introduction to Mechanics and Symmetry: A Basic Exposition of Classical Mechanical Systems. (2nd ed), Springer, New York, (1999)

[12] Park, J., Chung, W.-K., Geometric Integration on Euclidean Group With Application to Articulated Multibody Systems. IEEE Trans. Rob. 21(5), 850-863 (2005)

[13] Hatze, H., A general myocybernetic control model of skeletal muscle. Biol. Cyber. 28, 143-157, (1978)

[14] Wilkie, D.R., The mechanical properties of muscle. Brit. Med. Bull. 12, 177-182, (1956)

[15] Hill, A.V.,The heat of shortening and the dynamic constants of muscle. Proc. Roy. Soc. B76, 136-195, (1938)

[16] Vukobratovic, M., Borovac, B., Surla, D., Stokic, D., Biped Locomotion: Dynamics, Stability, Control, and Applications. Springer, Berlin, (1990)

[17] Ivancevic, V., Ivancevic, T., Neuro-Fuzzy Associative Machinery for Comprehensive Brain and Cognition Modelling. Springer, Berlin, (2007)

[18] Saunders, D.J.: The Geometry of Jet Bundles. Lond. Math. Soc. Lect. Notes Ser. 142, Cambr. Univ. Pr., (1989) 
[19] Massa, E., Pagani, E., Jet bundle geometry, dynamical connections and the inverse problem of Lagrangian mechanics. Ann. Inst. Henri Poincaré 61, 17, (1994)

[20] Giachetta, G., Mangiarotti, L., Sardanashvily, G., New Lagrangian and Hamiltonian Methods in Field Theory, World Scientific, Singapore, (1997)

[21] Sardanashvily, G.: Hamiltonian time-dependent mechanics. J. Math. Phys. 39, 2714, (1998)

[22] Mangiarotti, L., Obukhov, Yu., Sardanashvily, G., Connections in Classical and Quantum Field Theory. World Scientific, Singapore, (1999)

[23] Sardanashvily, G., Gauge Theory in Jet Manifolds. Hadronic Press, Palm Harbor, FL, (1993)

[24] R.S. Hamilton, Three-manifolds with positive Ricci curvature, J. Diff. Geom. 17, 255$306,(1982)$

[25] R.S. Hamilton, Four-manifolds with positive curvature operator, J. Dif. Geom. 24), $153-179,(1986)$

[26] R.S. Hamilton, The Ricci flow on surfaces, Cont. Math. 71, 237-261, (1988)

[27] R.S. Hamilton, The Harnack estimate for the Ricci flow, J. Dif. Geom. 37, 225-243, (1993)

[28] R.S. Hamilton, Non-singular solutions of the Ricci flow on three-manifolds, Comm. Anal. Geom. 7(4), 695-729, (1999)

[29] J. Milnor, Towards the Poincaré Conjecture and the Classification of 3-Manifolds, Not. Am. Math. Soc. 50(10), 1226-1233, (2003)

[30] G. Perelman, The entropy formula for the Ricci flow and its geometric applications, arXiv:math.DG/0211159, (2002)

[31] M.T. Anderson, Geometrization of 3-manifolds via the Ricci flow, Not. Am. Math. Soc. 51(2), 184-193, (2004) 